\title{
Solving Fractional Dynamical System with Freeplay by Combining Memory-Free Approach and Precise Integration Method
}

\author{
Q. X. Liu, X. S. He, J. K. Liu, Y. M. Chen, and L. C. Huang \\ Department of Mechanics, Sun Yat-sen University, No. 135 Xingang Road, Guangzhou 510275, China \\ Correspondence should be addressed to L. C. Huang; hlinch@mail.sysu.edu.cn
}

Received 10 December 2015; Revised 14 March 2016; Accepted 20 March 2016

Academic Editor: Laura Gardini

Copyright (c) 2016 Q. X. Liu et al. This is an open access article distributed under the Creative Commons Attribution License, which permits unrestricted use, distribution, and reproduction in any medium, provided the original work is properly cited.

The Yuan-Agrawal (YA) memory-free approach is employed to study fractional dynamical systems with freeplay nonlinearities subjected to a harmonic excitation, by combining it with the precise integration method (PIM). By the YA method, the original equations are transformed into a set of first-order piecewise-linear ordinary differential equations (ODEs). These ODEs are further separated as three linear inhomogeneous subsystems, which are solved by PIM together with a predictor-corrector process. Numerical examples show that the results by the presented method agree well with the solutions obtained by the RungeKutta method and a modified fractional predictor-corrector algorithm. More importantly, the presented method has higher computational efficiency.

\section{Introduction}

Recently, fractional derivative (FD) has been widely investigated because such mathematical model has merits over integer derivative in describing complex behaviors of some real systems. It has been confirmed that FD can model the realistic phenomena arising in various disciplines such as physics [1], materials science [2], solid mechanics [3], mechanical vibrations [4], biology [5], economics [6], and control theory [7].

Many numerical solution techniques were developed to obtain analytical, semianalytical, and/or numerical solutions of fractional dynamical systems, for example, the finite difference method $[8,9]$, predictor-corrector approach [10, 11], operational matrix method [12, 13], variational iteration method [14, 15], homotopy perturbation method [16, 17], Adomian's decomposition method [18, 19], to mention a few. Due to the nonlocal character of the fractional derivative, storing the past responses requires a large amount of computer memory. Moreover, a large amount of computational resources is spent on repeat processing of the convolution that describes FD. Accordingly, it is cumbersome to search for even numerical solutions of fractional dynamical systems in a long time duration.

In order to eliminate the drawback of long memory requirement, Yuan and Agrawal proposed a nonclassical approach [20]. In their scheme, the fractional differential equation can be converted into a set of first-order ordinary differential equations which can be solved by the RungeKutta (RK) method or the trapezoidal integration rule, and so forth. As there are no convolutions in the converted system, this method is called the Yuan-Agrawal (YA) memory-free approach. Later, Singh and Chatterjee [21] extended the memory-free principle introduced in the YA method, which can improve the computation accuracy.

It is worthy of noting that a troublesome problem in a freeplay model lies in determining switching points. Though the switching points can be approximated as the step length is chosen to be refined enough, the computation is very inefficient because refining the time step will lead to exponentially increasing computation cost. Therefore, it is necessary and worthwhile to propose some more efficient approaches to tackle this problem. 


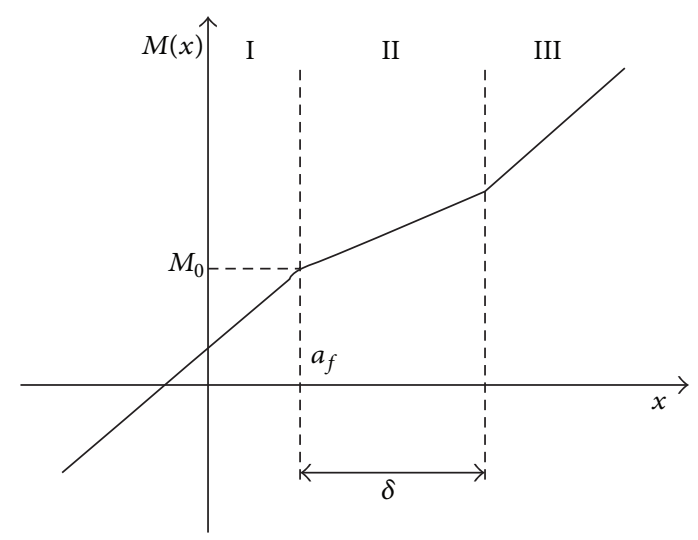

FIGURE 1: Sketch of a freeplay nonlinearity.

The precise integration method (PIM) initiated by Zhong and Williams [22] has been widely applied to various problems modeled by ordinary differential equations such as structural dynamics, optimal control, and flexible multibody dynamics problems $[23,24]$. This method is famous for its high accuracy and computation efficiency. We are motivated by its high precision and efficiency to apply this technique to Bagley-Torvik equation [2] with a piecewise linearity. A predictor-corrector technique will be applied to determine the time for switching from one subsystem to another subsystem. A modified fractional predictor-corrector (MFPC) method [25] will be utilized to validate the presented scheme. Numerical examples show that very accurate numerical results can be provided. Moreover, it is much more efficient than the MFPC or the YA method with RK scheme.

\section{Mathematical Model}

2.1. Equations of Motions. Considering the freeplay nonlinearity of a nonlinear spring, the dynamic equation of a single-degree-of-freedom spring-mass-damping system with fractional derivative is described as

$$
m D^{2} x(t)+c D^{\alpha} x(t)+k M(x)=f \sin (\omega t),
$$

where $m, c$, and $k$ denote the mass, damping coefficient, and stiffness, respectively, $D^{\alpha} x(t), 0<\alpha<1$, is the derivative of order $\alpha$ of the displacement function $x(t)$, and $f \sin (\omega t)$ is the externally applied force. Figure 1 shows the sketch of the nonlinear stiffness, $M(x)$, which is a freeplay nonlinearity usually referred to as a bilinear nonlinearity [26]:

$$
\begin{aligned}
& M(x) \\
& \quad= \begin{cases}M_{0}+x-a_{f}, & x<a_{f} \\
M_{0}+M_{f}\left(x-a_{f}\right), & a_{f} \leq x \leq a_{f}+\delta \\
M_{0}+x-a_{f}+\delta\left(M_{f}-1\right), & x>a_{f}+\delta\end{cases}
\end{aligned}
$$

where $M_{0}, a_{f}, M_{f}$, and $\delta$ are constants.

Under the transformation of a nondimensional time scale $\tau=\omega t$, (1) can be transformed into the following equation containing the fractional derivative with respect to nondimensional time $\tau$ :

$$
m \omega^{2} D^{2} x(\tau)+c \omega^{\alpha} D^{\alpha} x(\tau)+k M(x)=f \sin \tau .
$$

By means of gamma function and Laguerre integral formula, the fractional derivative term in (3) can be eliminated, and it becomes

$$
\begin{aligned}
& m \omega^{2} D^{2} x(\tau)+c \omega^{\alpha} \mu \int_{0}^{\infty} \varphi(y, \tau) d y+k M(x) \\
& =f \sin \tau
\end{aligned}
$$

where $\varphi(y, \tau)$ and $\mu$ are introduced variables as

$$
\begin{aligned}
\varphi(y, \tau) & =y^{2 \alpha-1} \int_{0}^{\tau} e^{-(s-\tau) y^{2}} D x(s) d s, \\
\mu & =\frac{2 \sin (\pi \alpha)}{\pi} .
\end{aligned}
$$

Taking derivative of (5) with respect to $\tau$, we can get

$$
D \varphi(y, \tau)=y^{2 \alpha-1} D x(\tau)-y^{2 \alpha+1} \varphi(y, \tau) .
$$

The integral in (4) can be approximated using the Laguerre integral formula [20] as

$$
\int_{0}^{\infty} \varphi(y, \tau) d y \approx \sum_{i=1}^{n} w_{i} e^{y_{i}} \varphi\left(y_{i}, \tau\right)
$$

where $w_{i}$ and $y_{i}(i=1, \ldots, n)$ are the Laguerre weights and node points, respectively. Therefore, (4) and (7) can be written as a set of first-order ordinary differential equations as

$$
\begin{aligned}
D x(\tau)= & \nu(\tau), \\
D \nu(\tau)= & \frac{1}{m \omega^{2}} f \sin (\tau)-\frac{k}{m \omega^{2}} M(x) \\
& -\frac{c \omega^{\alpha} \mu}{m \omega^{2}} \sum_{i=1}^{n} w_{i} e^{y_{i}} \varphi\left(y_{i}, \tau\right)
\end{aligned}
$$

with

$$
D \varphi\left(y_{i}, \tau\right)=y_{i}^{2 \alpha-1} \nu(\tau)-y^{2} \varphi\left(y_{i}, \tau\right), \quad i=1,2, \ldots, n
$$

Define $\mathbf{X}=\left[x, v, \varphi\left(y_{1}, \tau\right), \ldots, \varphi\left(y_{n}, \tau\right)\right]^{T}$, and (9) to (10) can be rewritten as the matrix equations

$$
\mathbf{X}^{\prime}= \begin{cases}\mathbf{A}_{1} \mathbf{X}+\frac{1}{m \omega^{2}} \mathbf{F} \sin \tau+\mathbf{C}_{1}, & x<a_{f} \\ \mathbf{A}_{2} \mathbf{X}+\frac{1}{m \omega^{2}} \mathbf{F} \sin \tau+\mathbf{C}_{2}, & a_{f} \leq x \leq a_{f}+\delta \\ \mathbf{A}_{3} \mathbf{X}+\frac{1}{m \omega^{2}} \mathbf{F} \sin \tau+\mathbf{C}_{3}, & x>a_{f}+\delta,\end{cases}
$$

where $\mathbf{A}_{i}, \mathbf{C}_{i}(i=1,2,3)$, and $\mathbf{F}$ are constant matrixes and the superscript denotes derivative with respect to nondimensional time $\tau$. 
For further simplification and convenience, we expand the dimension by adding two auxiliary variables $\xi_{1}(\tau)=$ $\cos (\tau)$ and $\xi_{2}(\tau)=\sin (\tau)$ satisfying [27]

$$
\begin{aligned}
& \xi^{\prime}=-\xi_{2}, \\
& \xi_{2}^{\prime}=\xi_{1} .
\end{aligned}
$$

Rewrite $\mathbf{X}=\left[x, v, \varphi\left(y_{1}, \tau\right), \ldots, \varphi\left(y_{n}, \tau\right), \xi_{1}, \xi_{2}\right]^{T}$, and (11) becomes

$$
\mathbf{X}^{\prime}=\mathbf{B}_{i} \mathbf{X}+\mathbf{C}_{i}, \quad i=1,2,3,
$$

where $\mathbf{B}_{i}$ is the coefficient matrix of subsystem $i(i=1,2,3)$ and $\mathbf{C}_{i}$ is a constant matrix.

According to the theories of the ordinary differential equations, the analytical solution of (13) can be given as

$$
\begin{aligned}
\mathbf{X}(\tau)= & \exp \left[\left(\tau-\tau_{0}\right) \cdot \mathbf{B}_{i}\right] \cdot \mathbf{X}\left(\tau_{0}\right) \\
& +\int_{\tau_{0}}^{\tau} \exp \left[(\tau-s) \cdot \mathbf{B}_{i}\right] \cdot \mathbf{C}_{i} d s \\
= & \exp \left[\left(\tau-\tau_{0}\right) \cdot \mathbf{B}_{i}\right] \cdot \mathbf{X}\left(\tau_{0}\right)+\mathbf{B}_{i}^{-1} \\
& \cdot\left\{\exp \left[\left(\tau-\tau_{0}\right) \cdot \mathbf{B}_{i}\right]-\mathbf{I}\right\} \cdot \mathbf{C}_{i} .
\end{aligned}
$$

The initial conditions for $\mathbf{X}(0)$ are given as $x(0)=x_{0}, \nu(0)=$ $\nu_{0}, \varphi\left(y_{i}, 0\right)=0(i=1, \ldots, n), \xi_{1}(0)=1$, and $\xi_{2}(0)=0$. Once a time step $(\Delta \tau)$ is chosen, the solutions for a given time series denoted as $[0, \Delta \tau, 2 \Delta \tau, \ldots, N \Delta \tau]$ can be generated. For example, at the $n$th time point, the solution is

$$
\begin{aligned}
\mathbf{X}(n \Delta \tau)= & \exp \left(n \Delta \tau \cdot \mathbf{B}_{i}\right) \cdot \mathbf{X}\left(\tau_{0}\right)+\mathbf{B}_{i}^{-1} \\
& \cdot\left[\exp \left(n \Delta \tau \cdot \mathbf{B}_{i}\right)-\mathbf{I}\right] \cdot \mathbf{C}_{i} \\
= & {\left[\exp \left(\Delta \tau \cdot \mathbf{B}_{i}\right)\right]^{n} \cdot \mathbf{X}\left(\tau_{0}\right)+\mathbf{B}_{i}^{-1} } \\
& \cdot\left\{\left[\exp \left(\Delta \tau \cdot \mathbf{B}_{i}\right)\right]^{n}-\mathbf{I}\right\} \cdot \mathbf{C}_{i} .
\end{aligned}
$$

Thus, the key to solve (13) is the computation of the exponential matrix for one time step, denoted as $\mathbf{T}=\exp \left(\Delta \tau \cdot \mathbf{B}_{i}\right)$.

2.2. Precise Integration Method. In the precise integration method (PIM), there is a simple yet efficient algorithm to compute the exponential matrix. The small time step, $\Delta \tau$, is further split uniformly as $\eta=\Delta \tau / 2^{n}$ with $n$ as large positive number (usually as 20 in real practice). By this means, the exponential matrix can be calculated recursively by

$$
\mathbf{T}=\exp \left(\Delta \tau \cdot \mathbf{B}_{i}\right)=\exp \left(\frac{\mathbf{B}_{i} \cdot \Delta \tau}{2^{n}}\right)^{2^{n}}=\exp \left(\mathbf{B}_{i} \cdot \eta\right)^{2^{n}} .
$$

Expanding $\exp \left(\mathbf{B}_{i} \cdot \eta\right)$ as a series and retaining several lowerorder terms,

$$
\begin{aligned}
\exp \left(\mathbf{B}_{i} \cdot \eta\right) \approx & \mathbf{I}+\mathbf{B}_{i} \cdot \eta+\frac{\left(\mathbf{B}_{i} \cdot \eta\right)^{2}}{2}+\frac{\left(\mathbf{B}_{i} \cdot \eta\right)^{3}}{6} \\
& +\frac{\left(\mathbf{B}_{i} \cdot \eta\right)^{4}}{24}=\mathbf{I}+\mathbf{T}_{a} .
\end{aligned}
$$

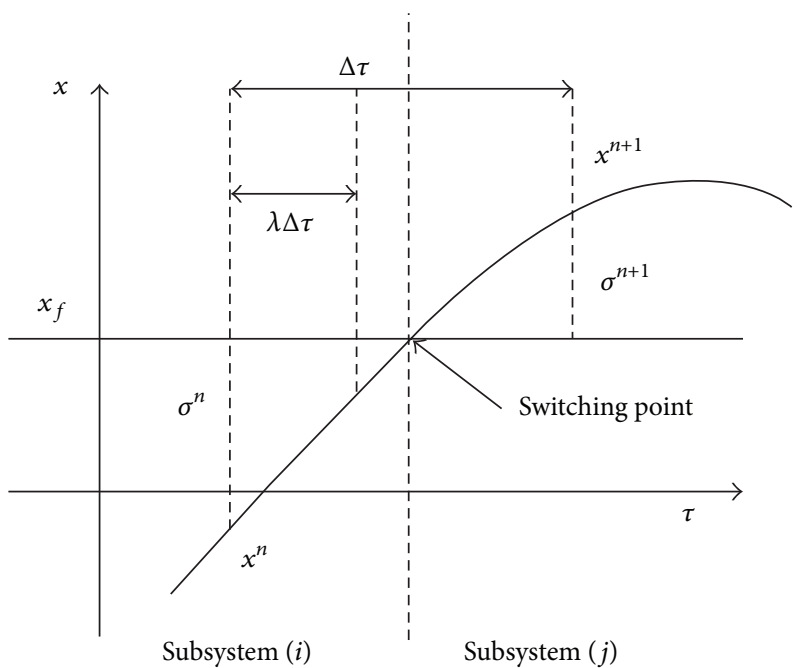

FIGURE 2: Illustration of the predictor-corrector algorithm for detecting the vibration state at a switching point.

The matrix $\mathbf{T}_{a}$ is introduced to distinguish the higher-order terms from I. Substitution of (17) into (16) results in

$$
\mathbf{T}=\left(\mathbf{I}+\mathbf{T}_{a}\right)^{2^{n}}=\left(\mathbf{I}+\mathbf{T}_{a}\right)^{2^{n-1}} \cdot\left(\mathbf{I}+\mathbf{T}_{a}\right)^{2^{n-1}} .
$$

The factorization should be iterated $n$ times, that is, for (iter $=$ 0 ; iter $<n$, iter ++$) \mathbf{T}_{a}=2 \mathbf{T}_{a}+\mathbf{T}_{a} \cdot \mathbf{T}_{a}$. After these iterations, the exponential matrix for one time step $(\Delta \tau)$ can be finally given as $\mathbf{T}=\mathbf{I}+\mathbf{T}_{a}$.

\subsection{A Predictor-Corrector Technique to Determine Switching} Points. Note that (13) is a piecewise-linear system consisting of three subsystems, and (15)-(18) are based on the fact that the vibration state remains to be located at the same subsystem. Generally, the state will finally leave one subsystem to another as the displacement $x$ passes through $x_{f}\left(x_{f}=a_{f}\right.$ or $a_{f}+\delta$ ), which indicates a switching point, as shown in Figure 2.

A predictor-corrector algorithm is applied to accurately find the time when the vibration state is passing one of the switching points. Denote $\sigma^{n}=x^{n}-x_{f}$ and introduce a ratio

$$
\lambda=\frac{\sigma^{n}}{\sigma^{n}-\sigma^{n+1}} .
$$

If $x^{n+1}$ is exactly at the switching point, we have $\sigma^{n+1}=0$ and $\lambda=1$. It is predicted that the time needed for $x^{n}$ to approach the switching point is about $\lambda \Delta \tau$. Then the predicted state is further corrected as

$$
\begin{aligned}
\mathbf{X}^{n+1}= & \exp \left(\lambda \Delta \tau \cdot \mathbf{B}_{i}\right) \cdot \mathbf{X}^{n}+\mathbf{B}_{i}^{-1} \\
& \cdot\left[\exp \left(\lambda \Delta \tau \cdot \mathbf{B}_{i}\right)-\mathbf{I}\right] \cdot \mathbf{C}_{i} \\
= & \mathbf{T}^{\lambda} \cdot \mathbf{X}^{n}+\mathbf{B}_{i}^{-1} \cdot\left(\mathbf{T}^{\lambda}-\mathbf{I}\right) \cdot \mathbf{C}_{i} .
\end{aligned}
$$

Repeating (19) and (20) until $\lambda$ approaches 1 closely enough with a very small tolerance error such as $10^{-12}$, the vibration state happening at switching points can be detected accurately and efficiently. 


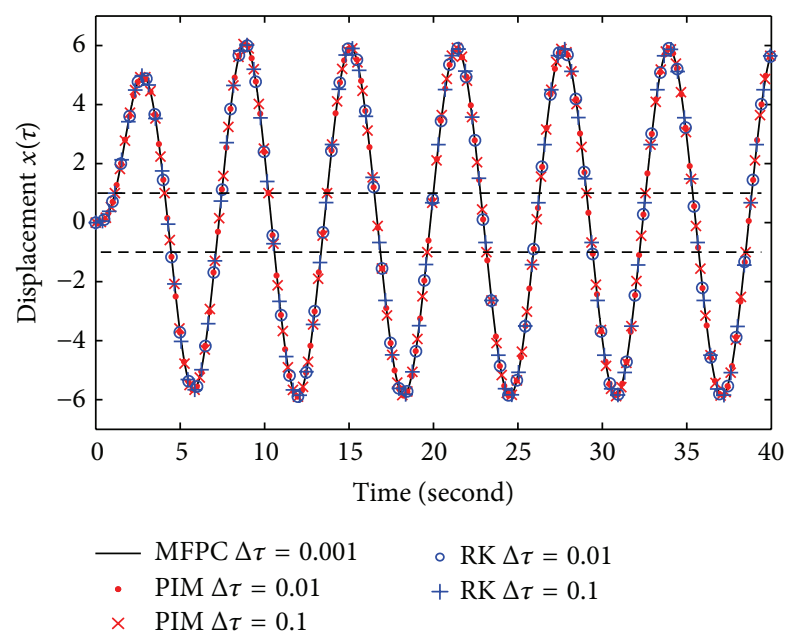

FIgURE 3: Displacements $x(\tau)$ obtained by PIM and RK.

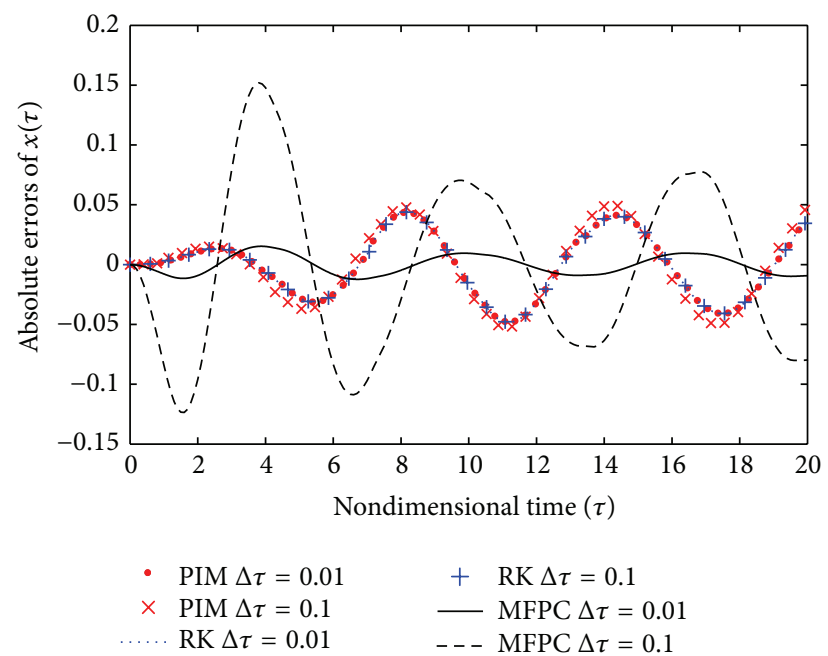

FIGURE 4: Absolute errors of the displacements $x(\tau)$ obtained by PIM, RK, and MFPC.

\section{Numerical Example}

3.1. A Single-Degree-of-Freedom Dynamical System. To validate the feasibility and accuracy of the presented algorithm, the parameters in (1) are chosen to be nondimensional values as $\alpha=0.5, m=1, c=1, k=1, \omega=1, M_{0}=1$, $a_{f}=-1, M_{f}=0, \delta=2$, and $f=5$. And the initial conditions are chosen as $x_{0}=\nu_{0}=0$. The results obtained by PIM and RK will be compared with those obtained by MFPC. And the results obtained by MFPC with a very small time step, $\Delta \tau=0.001$, are regarded as the standard solutions. Figure 3 shows the displacements $x(\tau)$ obtained by PIM and RK with the time step $\Delta \tau=0.1$ and 0.01 , respectively, when the number of Laguerre points is chosen as $n=30$. It can be seen that both the PIM and RK results agree well with the standard solutions.

In order to further check the accuracy, the absolute errors of the displacements obtained by PIM, RK, and MFPC with $\Delta \tau=0.1$ and 0.01 are shown in Figure 4. It shows that the

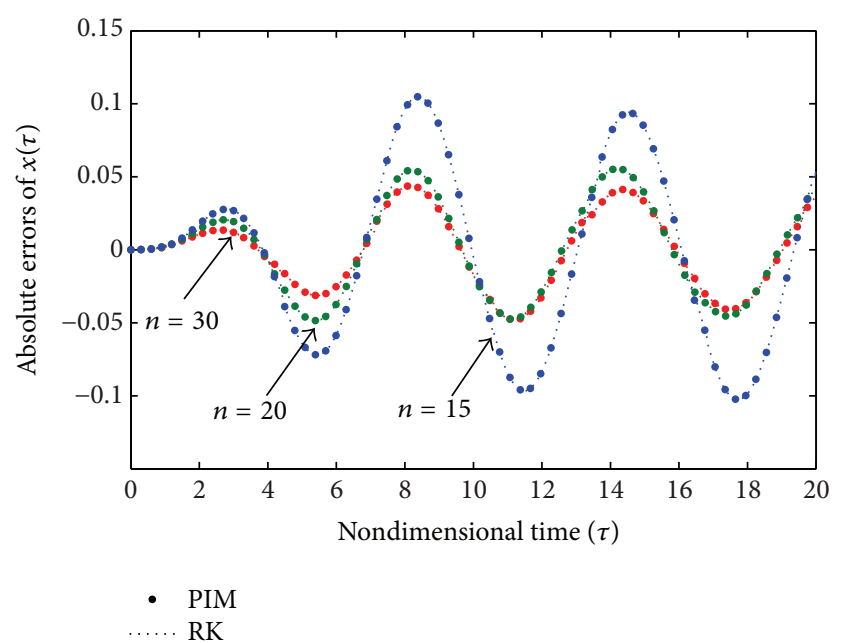

FIGURE 5: Comparison of absolute errors of displacements $x(\tau)$ obtained by PIM and RK for 15, 20, and 30 Laguerre node points.

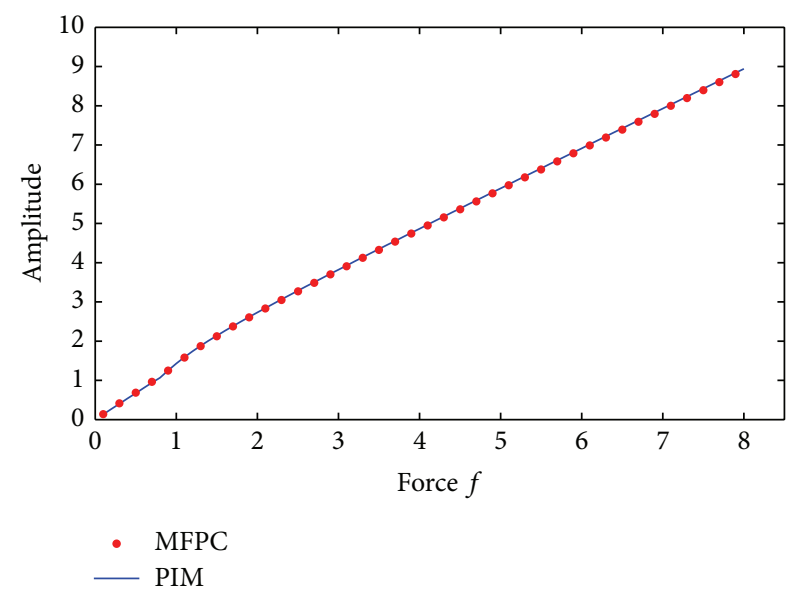

FIGURE 6: Comparison of the amplitudes versus the external force $f$ obtained by the MFPC and PIM.

results obtained by PIM and RK can reach almost the same accuracy. The accuracy of both methods should be about $1 \%$ for the amplitude of displacement. Figure 5 presents the absolute errors of results using PIM and RK for $\Delta \tau=0.01$ when the number of Laguerre points is chosen as $n=15$, 20 , and 30 , respectively. It clearly shows that the PIM and RK results converge as the number of Laguerre node points is increasing.

Figure 6 shows the characteristic of the amplitude of the displacement versus the external force amplitude $f$. Obviously, the amplitude is linearly related to the force amplitude. Furthermore, the amplitude-frequency characteristic of the system is also shown in Figure 7. The amplitude reaches its maximum when the frequency $\omega$ is close to 1.20 and then decreases as the frequency increases. It indicates a resonance as frequency is varying. By the way, both Figures 6 and 7 verify the accuracy and availability of the PIM results.

The computing times for the PIM, RK, and MFPC to calculate the responses of the system in 100 seconds are shown 
TABLE 1: Comparison of the computing times.

\begin{tabular}{lcccc}
\hline Number of Laguerre node points & Nondimensional time step & PIM (second) & RK (second) & MFPC (second) \\
\hline \multirow{2}{*}{$n=15$} & $\Delta \tau=0.1$ & 0.1217 & 13.76 & 4.3314 \\
& $\Delta \tau=0.01$ & 1.1055 & 24.05 & 381.05 \\
\hline \multirow{2}{*}{$n=20$} & $\Delta \tau=0.1$ & 0.1509 & 25.24 & 4.3314 \\
& $\Delta \tau=0.01$ & 1.2516 & 40.77 & 381.05 \\
\hline \multirow{2}{*}{$n=30$} & $\Delta \tau=0.1$ & 0.2551 & 59.85 & 7.3314 \\
& $\Delta \tau=0.01$ & 1.8333 & 380 & 381.05 \\
\hline
\end{tabular}

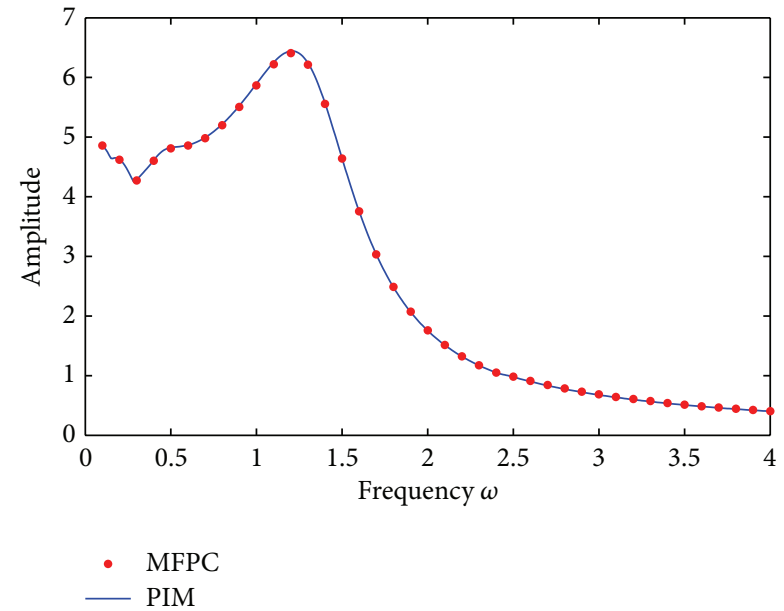

FIGURE 7: Comparison of the amplitude-frequency curve versus the external force $\omega$ obtained by the MFPC and PIM.

in Table 1. The computing times for PIM are much less than that for RK and MFPC. Moreover, the PIM computing times increase very slightly but the RK computing times roughly increase linearly with the increasing number of Laguerre node points.

3.2. A Two-Degrees-of-Freedom Dynamical System. In order to further examine the effectiveness, the presented algorithm is employed to solve a two-degrees-of-freedom dynamical system with fractional derivatives as

$$
\mathbf{M} D^{2} \mathbf{X}+\mathbf{C} D^{\alpha} \mathbf{X}+\mathbf{K X}+\mathbf{G}(\mathbf{X})=\mathbf{F} \cos (\omega t),
$$

where $\mathbf{M}, \mathbf{C}$, and $\mathbf{K}$ represent the mass, damping, and stiffness matrixes, respectively, $\mathbf{X}=\left[x_{1}, x_{2}\right]^{T}$ is the displacement matrix, $\mathbf{F}$ is the amplitude matrix of the applied harmonic force, and $\mathbf{G}(\mathbf{X})$ means the freeplay nonlinearity. While considering the freeplay nonlinearity of one degree of freedom, such as $x_{1}, \mathbf{G}(\mathbf{X})$ can be expressed as

$$
\begin{aligned}
& \mathbf{G}(\mathbf{X})=\left[\begin{array}{c}
g_{1}\left(x_{1}\right) \\
0
\end{array}\right], \\
& g_{1}\left(x_{1}\right)= \begin{cases}M_{0}+x_{1}-a_{f}, & x_{1}<a_{f} \\
M_{0}+M_{f}\left(x_{1}-a_{f}\right), & a_{f} \leq x_{1} \leq a_{f}+\delta \\
M_{0}+x_{1}-a_{f}+\delta\left(M_{f}-1\right), & x_{1}>a_{f}+\delta .\end{cases}
\end{aligned}
$$

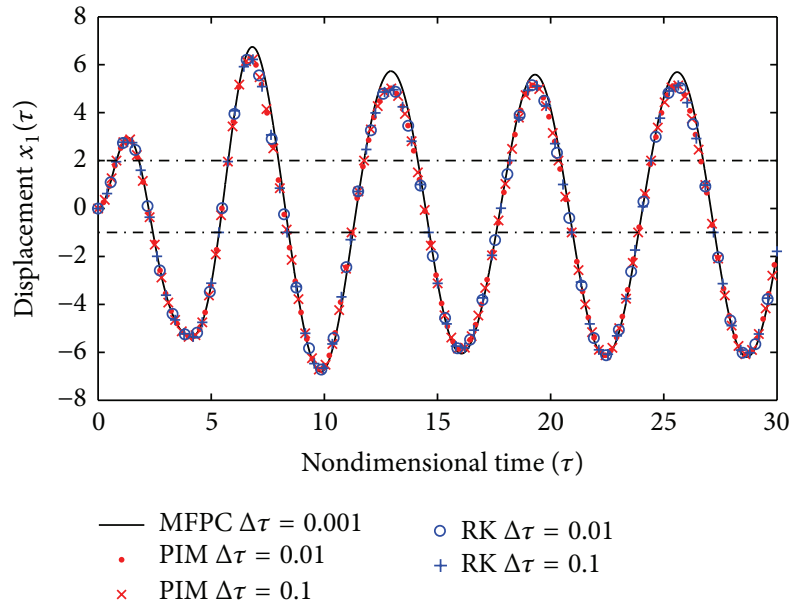

FIGURE 8: Displacements $x_{1}(\tau)$ for system (21) obtained by PIM and RK, respectively.

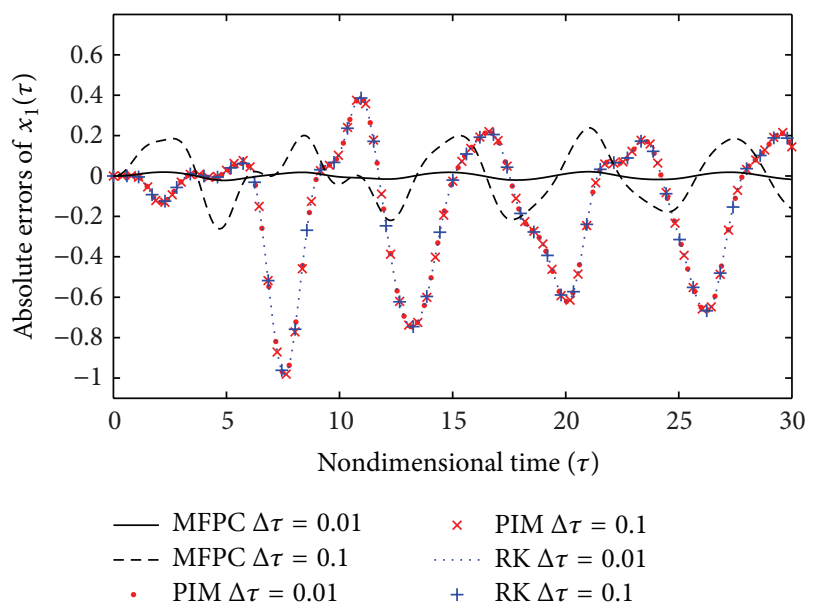

FIGURE 9: Absolute errors of the displacements $x_{1}(\tau)$ for system (21) obtained by PIM, RK, and MFPC, respectively.

In this case, the numerical values are chosen as $\mathbf{M}=\left[\begin{array}{ll}1 & 0 \\ 0 & 1\end{array}\right]$, $\mathbf{K}=\left[\begin{array}{cc}3 & -2 \\ -2 & 3\end{array}\right], \mathbf{C}=\left[\begin{array}{cc}0.6 & 0 \\ 0 & 0.6\end{array}\right], \mathbf{F}=\left[\begin{array}{c}10 \\ 0\end{array}\right], \alpha=0.5, \omega=1$, $M_{0}=1, a_{f}=-1, M_{f}=0.5$, and $\delta=3$. Choosing the number of Laguerre points as $n=30$, the time history curves of displacements $x_{1}$ obtained by MFPC, PIM, and LK with different time steps are shown in Figure 8. The PIM and RK results coincide very well with the MFPC results, respectively. Figure 9 shows the absolute errors of 
TABLE 2: Comparison of the computing times for solving system (2) over $[0,100]$.

\begin{tabular}{lcccc}
\hline Number of Laguerre node points & Nondimensional time step & PIM (second) & RK (second) & MFPC (second) \\
\hline \multirow{2}{*}{$n=10$} & $\Delta \tau=0.1$ & 0.1783 & 6.486 & 8.3587 \\
& $\Delta \tau=0.01$ & 1.3452 & 23.11 & 773.25 \\
\hline \multirow{2}{*}{$n=20$} & $\Delta \tau=0.1$ & 0.5004 & 27.90 & 8.3587 \\
& $\Delta \tau=0.01$ & 2.6063 & 32.62 & 773.25 \\
\hline \multirow{2}{*}{$n=30$} & $\Delta \tau=0.1$ & 0.9130 & 68.27 & 8.3587 \\
& $\Delta \tau=0.01$ & 4.3939 & 72.61 & 773.25 \\
\hline
\end{tabular}

displacements $x_{1}$ obtained by MFPC, PIM, and LK with $\Delta \tau=$ 0.1 and 0.01 , respectively, compared with the results obtained by MFPC with $\Delta \tau=0.001$. The attained PIM and RK results almost have the same accuracy.

Table 2 shows the comparison of the computing times of the MFPC, PIM, and RK upon calculating the responses of system (2) over $[0,100]$. Obviously, the PIM still possesses substantial advantages over the other two methods for highly dimensional systems.

\section{Conclusions}

This paper has presented an effective algorithm for solving the dynamic responses of fractional dynamical system with a freeplay nonlinearity. According to the YA memoryfree method, the system is transformed into a set of piecewise first-order ordinary differential equations without fractional derivative terms. And the transformed system is further separated into three subsystems. The switching points between subsystems are determined accurately by a predictor-corrector algorithm. Compared with the RungeKutta method and MFPC, the PIM is able to obtain very accurate solutions much more efficiently.

\section{Competing Interests}

The authors declare that they have no competing interests.

\section{Acknowledgments}

This work is supported by the National Natural Science Foundation of China (11572356, 111272361, and 51108472), Guangdong Province Natural Science Foundation (1414050000412), Guangdong Provincial Science \& Technology Program of China (2015A020217004), and Fundamental Research Funds for the Central Universities (15lgzd01).

\section{References}

[1] R. Hilfer, Applications of Fractional Calculus in Physics, World Scientific, Singapore, 2000.

[2] P. J. Torvik and R. L. Bagley, "On the appearance of the fractional derivative in the behavior of real materials," Journal of Applied Mechanics, vol. 51, no. 2, pp. 294-298, 1984.

[3] Y. A. Rossikhin and M. V. Shitikova, "Application of fractional calculus for dynamic problems of solid mechanics: novel trends and recent results," Applied Mechanics Reviews, vol. 63, no. 1, pp. 010801-1-010801-52, 2010.

[4] M. S. Tavazoei, M. Haeri, M. Attari, S. Bolouki, and M. Siami, "More details on analysis of fractional-order Van der Pol oscillator," Journal of Vibration and Control, vol. 15, no. 6, pp. 803-819, 2009.

[5] R. L. Magin, Fractional Calculus in Bioengineering, Begell House, Redding, Calif, USA, 2006.

[6] M. S. Abd-Elouahab, N.-E. Hamri, and J. Wang, "Chaos control of a fractional-order financial system," Mathematical Problems in Engineering, vol. 2010, Article ID 270646, 18 pages, 2010.

[7] I. Podlubny, Fractional Differential Equations: An Introduction to Fractional Derivatives, Fractional Differential Equations, to Methods of Their Solution and Some of Their Applications, Academic Press, San Diego, Calif, USA, 1998.

[8] M. M. Meerschaert and C. Tadjeran, "Finite difference approximations for fractional advection-dispersion flow equations," Journal of Computational and Applied Mathematics, vol. 172, no. 1, pp. 65-77, 2004.

[9] J. Huang, Y. Tang, L. Vázquez, and J. Yang, "Two finite difference schemes for time fractional diffusion-wave equation," Numerical Algorithms, vol. 64, no. 4, pp. 707-720, 2013.

[10] K. Diethelm, N. J. Ford, and A. D. Freed, "A predictor-corrector approach for the numerical solution of fractional differential equations," Nonlinear Dynamics, vol. 29, no. 1-4, pp. 3-22, 2002.

[11] W. Deng, "Short memory principle and a predictor-corrector approach for fractional differential equations," Journal of Computational and Applied Mathematics, vol. 206, no. 1, pp. 174-188, 2007.

[12] A. Saadatmandi and M. Dehghan, "A new operational matrix for solving fractional-order differential equations," Computers and Mathematics with Applications, vol. 59, no. 3, pp. 1326-1336, 2010.

[13] P. Mokhtary, F. Ghoreishi, and H. M. Srivastava, “The MüntzLegendre Tau method for fractional differential equations," Applied Mathematical Modelling, vol. 40, no. 2, pp. 671-684, 2016.

[14] Z. M. Odibat and S. Momani, "Application of variational iteration method to nonlinear differential equations of fractional order," International Journal of Nonlinear Sciences and Numerical Simulation, vol. 7, no. 1, pp. 27-34, 2006.

[15] E. Hesameddini, A. Rahimi, and E. Asadollahifard, "On the convergence of a new reliable algorithm for solving multi-order fractional differential equations," Communications in Nonlinear Science and Numerical Simulation, vol. 34, pp. 154-164, 2016.

[16] S. Abbasbandy, "Homotopy perturbation method for quadratic Riccati differential equation and comparison with Adomian's decomposition method," Applied Mathematics and Computation, vol. 172, no. 1, pp. 485-490, 2006. 
[17] A. A. Elbeleze, A. Kılıçman, and B. M. Taib, "Homotopy perturbation method for fractional Black-Scholes European option pricing equations using Sumudu transform," Mathematical Problems in Engineering, vol. 2013, Article ID 524852, 7 pages, 2013.

[18] S. Momani and Z. Odibat, "Analytical solution of a timefractional Navier-Stokes equation by Adomian decomposition method," Applied Mathematics and Computation, vol. 177, no. 2, pp. 488-494, 2006.

[19] C. Li and Y. Wang, "Numerical algorithm based on Adomian decomposition for fractional differential equations," Computers and Mathematics with Applications, vol. 57, no. 10, pp. 1672-1681, 2009.

[20] L. Yuan and O. P. Agrawal, "A numerical scheme for dynamic systems containing fractional derivatives," Journal of Vibration and Acoustics, vol. 124, no. 2, pp. 321-324, 2002.

[21] S. J. Singh and A. Chatterjee, "Galerkin projections and finite elements for fractional order derivatives," Nonlinear Dynamics, vol. 45, no. 1-2, pp. 183-206, 2006.

[22] W. X. Zhong and F. W. Williams, "Precise time step integration method," Proceedings of the Institution of Mechanical Engineers, Part C: Journal of Mechanical Engineering Science, vol. 208, no. 6, pp. 427-430, 1994.

[23] Y. X. Gu, B. S. Chen, H. W. Zhang, and Z. Guan, "Precise timeintegration method with dimensional expanding for structural dynamic equations," AIAA Journal, vol. 39, no. 12, pp. 23942399, 2001.

[24] Y. M. Chen and J. K. Liu, "Nonlinear aeroelastic analysis of an airfoil-store system with a freeplay by precise integration method," Journal of Fluids and Structures, vol. 46, pp. 149-164, 2014.

[25] Q. X. Liu, J. K. Liu, and Y. M. Chen, "Initial conditionsindependent limit cycles of a fractional-order van der Pol oscillator," Journal of Vibration and Control, 2015.

[26] B. H. K. Lee, S. J. Price, and Y. S. Wong, "Nonlinear aeroelastic analysis of airfoils: bifurcation and chaos," Progress in Aerospace Sciences, vol. 35, no. 3, pp. 205-334, 1999.

[27] P. J. Davis and I. Polonsky, "Numerical interpolation, differentiation, and integration," in Handbook of Mathematical Functions, chapter 25, Dover, New York, NY, USA, 1972. 


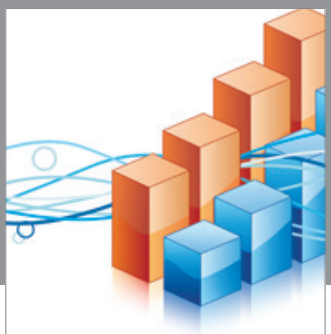

Advances in

Operations Research

vatem alat4

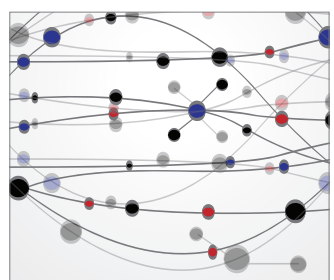

\section{The Scientific} World Journal
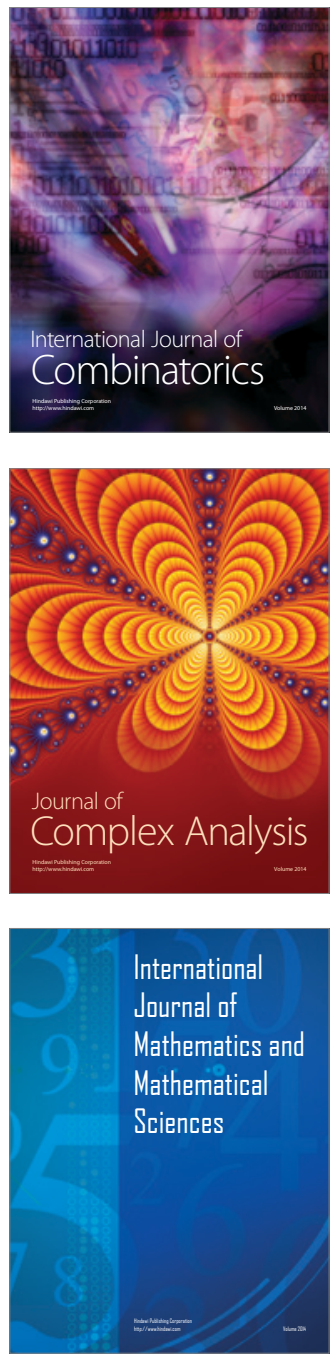
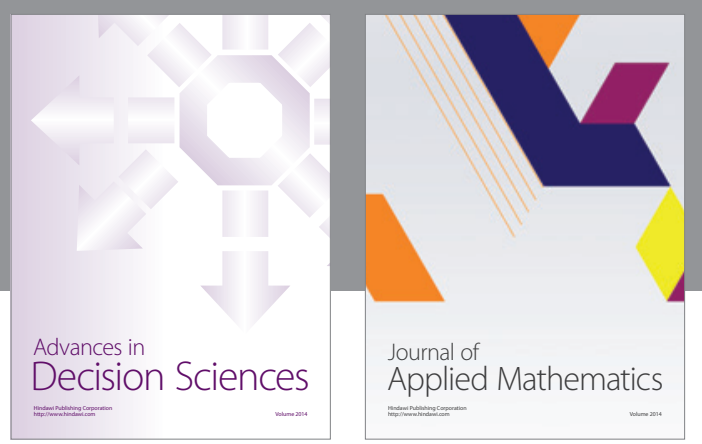

Algebra

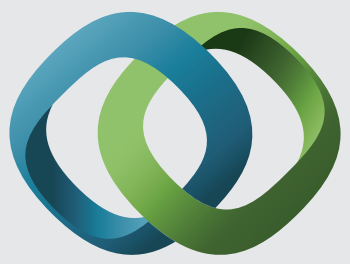

\section{Hindawi}

Submit your manuscripts at

http://www.hindawi.com
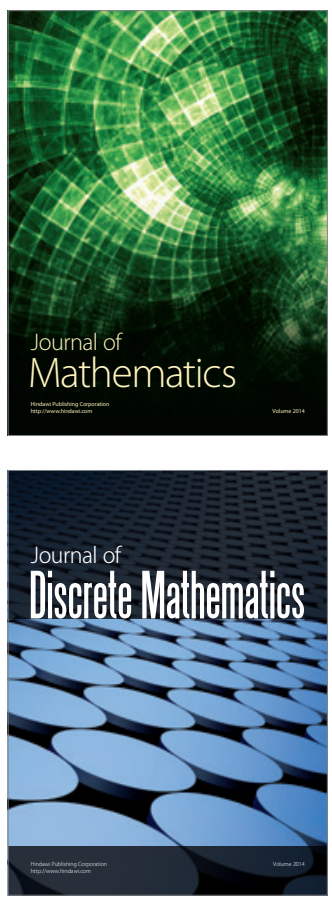

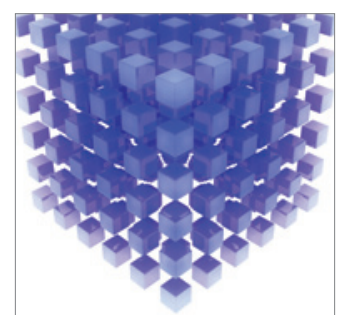

Mathematical Problems in Engineering
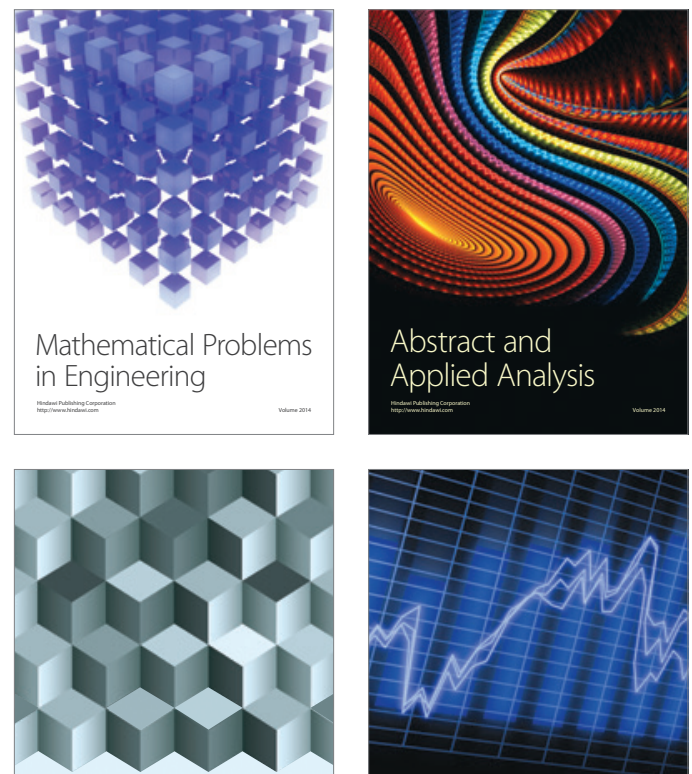

Journal of

Function Spaces

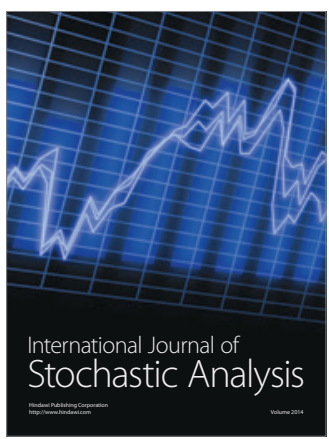

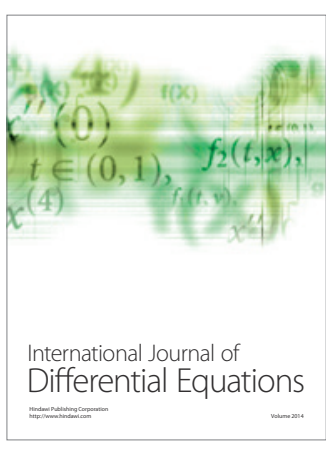
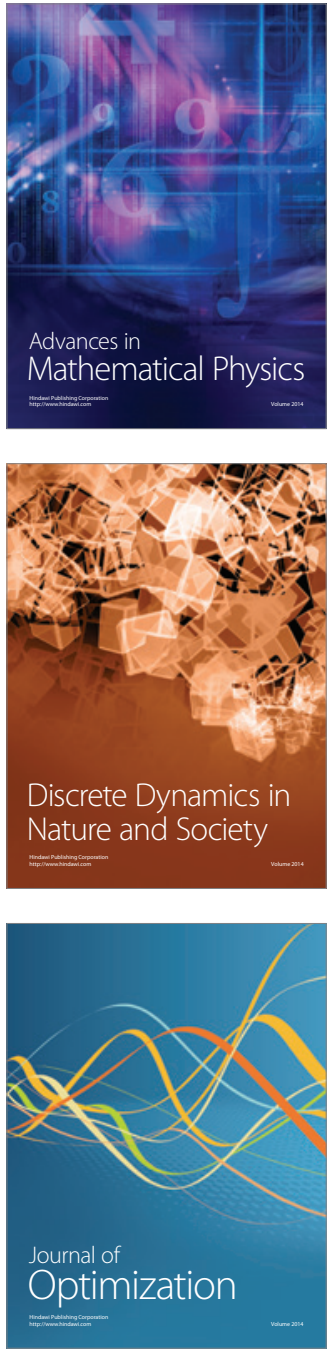